\title{
Actualidad en teoría de la historia. Una mirada desde las "relaciones con el pasado"
}

Current historical theory. A view from the "relationships with the past"

\author{
Daniel Ovalle Pastén \\ Universidad Andrés Bello \\ Universidad de Valparaíso \\ ovalle.daniel@gmail.com
}

\begin{abstract}
Resumen
El artículo es un repaso sintético por la actualidad de las discusiones en teoría de la historia desde tres ejes temáticos. En la introducción, se problematiza qué se entiende hoy por teoría de la historia; luego, en el segundo apartado se desarrolla cuáles son sus principales tendencias; y por último, se discute la noción de "relaciones con el pasado" del teórico Herman Paul para argumentar que la teoría de la historia cumple un trabajo relevante a la hora de comprender la propia historicidad de la disciplina.
\end{abstract}

Palabras clave: teoría de la Historia, relaciones con el pasado, historicidad.

\begin{abstract}
The article is a synthetic review of the current discussions of historical theory from three thematic axes. In the introduction, it problematizes what is understood today by historical theory, then in the second section it develops what the main tendencies are and, finally, it discusses the notion "relationships with the past" of the theorist Herman Paul to argue that the historical theory fulfills a relevant work when it comes to understanding the own historicity of the discipline.
\end{abstract}

Keywords: theory of history, relations with the past, historicity. 


\section{Introducción: ¿qué teoría de la Historia?}

La teoría de la Historia se encuentra en un momento disciplinar alentador gracias a un gran número de encuentros académicos, redes de trabajo, nuevas revistas y libros que se vienen generando en los últimos años, aunque también es cierto que todavía sigue relegada a espacios muy limitados dentro de la disciplina historiadora. Las razones de este problema pueden ser muchas y no es el objetivo de este trabajo analizarlas. Al respecto es altamente recomendable la lectura en línea de un reciente manifiesto titulado Theses on Theory and History, de los autores Ethan Kleinberg, Joan Wallach Scott y Gary Wilder. Si bien no compartimos algunas de las tesis allí esgrimidas, el texto es claro en orientar la discusión del rechazo a los problemas teóricos por parte de la mayoría de los historiadores (http://theoryrevolt.com/). Las múltiples desconfianzas generadas primero por las filosofías de la historia teleológicas y luego por las malas interpretaciones (y poca recepción crítica) del narrativismo que comenzaba a ganar terreno desde que Hayden White publicara Metahistoria en 1973, todavía resuenan en los pasillos de las escuelas de Historia en buena parte del mundo. Muchos académicos siguen creyendo en la "miseria de la teoría" o dan crédito en sus clases y escritos al desmedro de lo teórico al modo de los primeros Annales. Lo cierto es que el intercambio entre historiadores y filósofos o teóricos de la Historia todavía parece ser un diálogo de sordos, aunque hay razones para ser optimistas.

Estamos confiados en que esto puede cambiar en la medida que la disciplina se entere de que la teoría de la Historia hace ya bastante años ha dejado de posicionarse como un ente normativo. Los teóricos y filósofos de la Historia ya no siguen creyendo que sus investigaciones sirvan para nada más que para la propia comprensión de una disciplina que ha sido, es y seguirá siendo histórica; de lo contrario nuestro trabajo no tendría razón de ser. Esto quiere decir que la escritura de la Historia es histórica a la vez (responde a su lugar de enunciación), y que la teoría de la Historia que representamos trabaja desde la autorreferencialidad de la propia operación historiográfica. En otras palabras, no puede sino posicionarse a posteriori de la representación historiadora para intentar develar la propia historicidad de su desarrollo en atención a los cambios sociales y con ellos, al devenir de la ciencia en general. Desde la recepción de los postulados de Michel De Certeau, el historiador mexicano Alfonso Mendiola lo expresa de manera clara:

Nosotros no explicamos el pasado; explicamos observaciones sobre el pasado - o, más bien, explicamos el pasado sólo en la medida en que lo hemos considerado a la luz de algún tipo de descripción o especificación verbal. Como se puede ver, este nuevo enfoque de la escritura de la historia sólo es comprensible desde una postura reflexiva, pues exige que el investigador se pregunte por qué dice lo que dice el pasado, y deje de creer que el pasado se expresa sin su intervención (2000: 182).

Ya entrado el siglo XXI la situación de la teoría de la Historia ha cambiado en términos globales. A los aportes de clásicos del norte del mundo como Reinhart Koselleck, Jörn Rüsen, Chris Lorenz, Aviezer Tucker, Hayden White y Frank Ankersmit, se suman hoy jóvenes académicos como Herman Paul, Jouni-Matti Kuukkanen, Marek Tamm, Berber Bevernage, Zoltán Boldizsár Simon, Kalle Pihlainen y Ethan Kleinberg; sumado a los aportes de los clásicos en nuestra lengua como José Elías Palti, Fernando Betancourt, Jaume Aurell, Alfonso Mendiola y Guillermo 
Cermeño, a los cuales se añade otra generación de historiadores teóricos más jóvenes como Valdei Lopes de Araujo y Fernando Nicolazzi. Para el caso de nuestro país, es justo mencionar los trabajos de Pablo Aravena, Rodrigo Ahumada, Ignacio Muñoz Delaunoy, Luis de Mussy y Miguel Valderrama. Con todo, clásicos e iniciados en teoría de la Historia intentan proyectar investigaciones y reflexiones desde las dos grandes problemáticas que sustentan su quehacer, que a decir de una autoridad en la materia como Rüsen, son las condiciones de posibilidad en la conformación del conocimiento histórico (meta-teoría) y los enunciados teóricos acerca de las transformaciones temporales del ser humano en sociedad: el problema de la historicidad (2013:90).

Aportes recientes han llevado incluso a plantear la poca funcionalidad que tiene hoy separar las nociones teoría de la Historia y filosofía de la Historia. Es el caso de Berber Bevernage, quien aspira a superar las diferencias entre filosofía especulativa de la Historia versus filosofía crítica de la Historia, para confluir en una teoría de la Historia que, al incorporar el problema de la historicidad - la interpretación social del tiempo - en la construcción del conocimiento histórico, asuma el problema filosófico como algo obligatorio (2012: 113-114). Es en esta línea donde posicionamos este trabajo.

Consideramos que la valoración de la historicidad para la teoría de la Historia es de primer orden, toda vez que pensamos toda representación historiadora como una interpretación temporal que, por tanto, se proyecta hacia la valoración (o no) de un sentido. El problema del tiempo se transforma así en uno de los aspectos más relevantes para la teoría de la Historia actual, dificultad que ha sido largamente discutida sigue siéndolo - por filósofos, desde Aristóteles hasta nuestros días. El problema es que los historiadores, observa Chris Lorenz, - uno de los teóricos más respetados en la actualidad han sido prácticamente los últimos en sumarse a la discusión acerca del tiempo, algo que parece a todas luces un contrasentido. Los historiadores, explica, hicieron del tiempo algo invariable, una especie de estructura que adolece de historia (2017: 109-110). Al parecer, creyeron que la máxima de Marc Bloch "ciencia (...) de los hombres en el tiempo" (1952: 18) era una especie de invariable histórica que no estaba sujeta a investigación. Discusiones desde la filosofía del lenguaje como los tres tomos de Temps et récit (1983-1985) de Paul Ricoeur y los aportes de François Hartog en la historización del tiempo desde la existencia de "regímenes de historicidad" (desde la huella de Koselleck) están cambiando este verdadero atraso disciplinar. Los historiadores ya no tienen excusa: el tiempo no existe, existen múltiples temporalidades y nuestro deber es historizar las continuidades y cambios en las formas de construir la temporalidad, así como la teoría de la Historia debe dar cuenta de ese conocimiento para la disciplina. En este sentido, en el estudio de la historicidad en relación con la escritura de la Historia, explica François Dosse,

"el historiador debe pensar, más que nunca, la historicidad misma de su trabajo y la historicidad de toda categorización de las realidades sociales del pasado (... lo que) que conduce al historiador a una interrogación epistemológica e historiográfica más ambiciosa y más cargada de desafíos que antes" (2012: 14).

En definitiva, y siguiendo a Rüsen, se pueden observar los siguientes parámetros para comprender nuestra matriz disciplinar: a) la necesidad de orientación de las prácticas vivenciales humanas y que es la determinante en la elección de distintos intereses de conocimiento acerca del pasado; b) los horizontes dominantes 
sobre el pasado humano desde donde éste aparece como historia entre el presente y el futuro (conciencia histórica); c) el método histórico como prenda de cientificidad (narrativa y no nomológica); d) las distintas formas historiográficas desde donde el conocimiento histórico espera potenciales receptores; y e) la función de la historiografía en cada presente (2013: 96-97).

La historiografía es una práctica cultural que elabora simbólicamente el tiempo, la que sumada a otras prácticas sociales termina por formar las distintas conciencias históricas que los grupos humanos representan de forma colectiva (Rüsen, 2013: 47-84). Visto así, la teoría de la Historia no termina en el análisis textual. Pretende ir más allá para dar cuenta del sentido histórico del tiempo que los propios historiadores llevaron al papel. Por eso no puede sino problematizar la conciencia histórica como fenómeno inherente a la escritura de la Historia, validando la impronta de nuestra "matriz disciplinar", donde la teoría de la Historia debe dar cuenta de cada uno de los tópicos enunciados por Rüsen.

Así como existe relación entre temporalidad y escritura de la Historia, este trabajo toma nota, de forma complementaria, de los distintos tipos de "relaciones con el pasado", expresadas tanto por los sujetos en sociedad como por el sujeto historiador. En esto seguimos al joven teórico de la Historia Herman Paul, quien ha publicado el año 2015 un interesante libro introductorio a la teoría de la Historia desde una visión que entiende toda referencia al pasado como un tipo de relación. Siguiendo a Rüsen y Mark Day, Paul expresa que las distintas relaciones con el pasado son herramientas teóricas que dan cuenta de la complejidad del pensamiento histórico; ellas remiten desde un problema semántico al conjunto de dimensiones que los sujetos desarrollan para mirar - evocando a Ricoeur lo ya sido: en resumen, relaciones epistémicas, morales, políticas, estéticas y materiales. Como desarrollaremos, las primeras cuatro asumen una relación teleológica, ya que están referidas a eso que le pedimos al pasado; están atadas a propósitos desde un presente con respecto al pasado. En cambio, la relación material, explica Paul, no es teleológica, pues "gira en torno a lo que el pasado hace a las personas y no lo que las personas hacen con el pasado" (2015a: 34). Lo que nos interesa resaltar, algo no desarrollado en profundidad por Paul - y esto no es una crítica al texto pues no está dentro de los objetivos que el autor se plantea desarrollar - es que todos estos tipos de relaciones, las cuales nunca operan de forma independiente, corresponden a relaciones temporales entre pasado, presente y futuro.

En resumen, somos parte de una renovada forma de asumir la teoría de la Historia, entendida ahora como un campo reflexivo y epistemológico que, en palabras de Fernando Betancourt, describe reflexivamente los niveles que conforman su base disciplinaria y sus complejas interacciones, intentando mostrar las diversas formas operativas que conforman su lógica de investigación, los espacios sociales que posibilitan la operación historiográfica $y$, finalmente, los criterios que posibilitan su expansión discursiva (2011: 17).

\section{Teoría de la Historia hoy: una visión panorámica}

Las actuales discusiones acerca del trabajo teórico en historiografía están centradas en la superación de la polaridad entre empiristas y narrativistas (Paul, 2015b: 455), lo que para algunos ha devenido en una serie de teorías llamadas postnarrativistas (Kuukkanen, 2015). Extrapolando un poco el diagnóstico de Paul que por cierto compartimos - habrá que decir que esta disputa enfrenta al pensamiento moderno de la escritura de la Historia con el postmoderno. 
En un artículo reciente hemos interpretado esta disputa de la mano de una lectura sistemática de los aportes teóricos de Roger Chartier y Paul Ricoeur - que nuestra hipótesis de trabajo presenta totalmente complementarios - frente a los lineamientos del narrativismo postmoderno (Ovalle, 2018), por lo que no volveremos sobre lo allí desarrollado. Nada más decir que las posiciones modernas apelan a la necesidad de las condiciones de posibilidad del conocimiento histórico, lo que viene bien entenderlo desde la noción ricoeuriana de "pacto de verdad" entre historiador y lector (Ovalle, 2017), que refiere a ese "realismo crítico" asociado al escrutinio del testimonio y el documento como fundamento de la metodología histórica que acredita la representación científica del pasado bajo la confianza de una teoría por correspondencia indirecta (Ricoeur, 2000: 363).

Por otro lado, las posturas narrativistas - desde White y un primer momento de Ankersmit - y las actuales "teorías de la presencia" (segundo momento de Ankersmit, Eelco Runia y otros) desprecian el valor de la epistemología ya que centran las relaciones con el pasado desde un plano estético. La posición postmoderna desde el constructivismo narrativo radical, representada en los trabajos de Hayden White, Frank Ankersmit y Keith Jenkins, desacredita la epistemología de la historia y la racionalización de sus bases operacionales en el proceso de consecución del conocimiento histórico. Este último, si bien indiciario como dijera Carlo Ginzburg, funciona apegado a reglas en vías de explicar y comprender los acontecimientos pasados. Para los postmodernos, las representaciones del pasado no apelan a la verdad ni a la falsedad, sino que se enmarcan dentro aquello que se dice del pasado. Rüsen es claro al respecto:

La crítica decisiva a la racionalidad metodológica teórica en las ciencias de la historia confluye con corrientes de la teoría de la historia en el ámbito del pensamiento postmoderno, que pone en tela de juicio precisamente aquellas concepciones que fundamentan las ciencias de la historia como disciplina especializada: la concepción de la historia definible como ámbito de experiencia con un procedimiento cognitivo particular que establece una calidad temporal y la cientificidad del pensamiento histórico. La "historia" se critica como producto de una formación de sentido narrativa acerca de la experiencia del tiempo cuyos aspectos decisivos son de índoles poética o retórica, es decir, se trata de aspectos que precisamente no requieren una racionalidad metodológica (Rüsen, 2013: 121)

Para Rüsen, el pensamiento postmoderno conduce a una "irracionalización" peligrosa que dificulta la "orientación histórica en las prácticas vivenciales humanas", obligando al teórico de la historia a pensar el problema del sentido del pasado estudiando, tanto para el presente como el futuro. En consecuencia, el historiador alemán argumenta que desde una postura postmoderna: "La verdad del conocimiento se reduciría a la belleza de la forma, y el efecto práctico del conocimiento histórico ya no obedecería al criterio de justificación racional, sino a la fuerza sugestiva de medios desenfrenados de la inmediatez estética" (2013: 133).

Se podría decir que estos son los dos grandes paradigmas en teoría de la Historia y que de la discusión de ambos hacia posturas proclives, antagónicas o consensuadas hoy se nos presenta un panorama complejo que por cierto no podemos sintetizar de manera cabal en estas páginas. Esa empresa podría ser fácilmente objeto de una tesis doctoral. Ahora bien, por una parte, están aquellos que defienden una teoría de la Historia científica desde el conocimiento probable (inferencias y modelos causales), los cuales se han desprendido del "sueño 
objetivista", pero que separan aguas absolutas del pensamiento narrativista y/o postmoderno. Su mayor exponente es Aviezer Tucker (2004, 2014). Podría denominárseles neo-objetivistas, pues defienden una teoría de la correspondencia al estilo rankeano, sin creer por ello que el pasado se puede representar "tal y como fue".

De las posiciones que dialogan con el narrativismo, presentamos dos subgrupos: los que validan los aportes del narrativismo, como es el caso de Herman Paul (2015) y Kalle Pihlainen (2014), y otros que desde el mismo diálogo critican la excesiva importancia dada por éstos al escrito histórico por sobre el pasado mismo, relegando los procesos cognitivos de creación de conocimiento histórico. En estos últimos, autores como Jouni-Matti Kuukkanen (2015) y Marek Tamm (2014), muy en diálogo con Rüsen, defienden una teoría de la historia racional y argumentativa. Son los llamados postnarrativistas, cuyos trabajos integradores se pueden posicionar con la última etapa de Ricoeur en lo referente a su filosofía de la Historia (llama la atención que en el libro de Kuukkanen no haya una sola referencia a Ricoeur). Nuestra propia posición en trabajos ya citados debe ser establecida en esta posición, aquella que dialoga con el narrativismo desde los innegables beneficios del giro lingüistico.

El caso de Kuukkanen es para destacar, pues desde un diálogo fructífero con las tesis más relevantes de White y Ankersmit es capaz de elaborar una "tercera vía", siempre desde una defensa al estatus epistémico de la operación historiográfica; por tanto, desde un compromiso por la búsqueda de aquello que realmente pasó, pero a sabiendas de que el discurso historiográfico está mediado irremediablemente por el lenguaje del presente de la enunciación. El resultado es una teoría no representacionalista del pasado (Kuukkanen, 2015: 50-67), pues para el profesor de la Universidad de Oulu, el discurso historiográfico es ante todo una defensa de hipótesis y argumentaciones racionales que no tienen relación directa con el pasado; he ahí su conexión directa con el narrativismo y constructivismo. Marek Tamm (2014) es también parte de este grupo, argumentando la existencia deun "regulativoidealdeobjetividad", situándose desde una posición pragmática, donde la verdad del discurso histórico dependerá de tres pasos interrelacionados: su fuerza ilocucionaria; el consenso disciplinar acerca del método, los valores cognitivos y las virtudes epistémicas; así como de la evidencia y prueba documental.

En un último grupo posicionamos a aquellos teóricos que han vuelto al problema ontológico esperando superar la visión narrativista, pero compartiendo a todas luces su ímpetu fundacional. Estos trabajos se posicionan desde la denominada "experiencia del pasado", problema que se debe asociar al desarrollo de las filosofías de la presencia. Entre ellos se encuentran el segundo momento de Ankersmit (2005) - su salida a esa "prisión del lenguaje" - con su tesis de la "experiencia sublime", Eelco Runia (2006), Ethan Kleinberg (2017) y Ranjan Ghosh (2013), quienes han puesto de relieve problemas estéticos y no lingüísticos del pasado en el presente, compartiendo con White el descrédito hacia la epistemología de la historia. En Sublime Historical Experience (publicado primeramente en inglés el año 2005), Ankersmit dirige la mirada al cómo nos relacionamos con el pasado desde el problema de la conciencia histórica, intentando de esta manera salir de aquella "camisa de fuerza" de la narratividad. Ankersmit se propuso mostrar cómo la experiencia del pasado llega a ser un problema para el historiador que espera al final del camino representar parte de ese pasado. La pregunta que guía su obra es:

Cómo y por qué hemos llegado a fascinarnos por nuestro pasado colectivo en términos de la noción "experiencia histórica sublime". Para una nación, una colectividad, una cultura o civilización 
que ha tenido tal experiencia histórica sublime, el pasado y la conciencia de ese pasado devienen realidades ineluctables. El pasado será constituyente de lo que son en la actualidad en la misma medida que nuestras extremidades son parte constituyente de nuestros cuerpos, y olvidar el pasado entonces sería una amputación intelectual (Ankersmit, 2005: 15).

Si bien es destacable el intento de Ankersmit de posicionar la conciencia histórica como un problema central para la teoría de la Historia, carece, creemos, de fundamentos sólidos al hacerlo desde una posición en extremo subjetivista. Para ello, no acude a nociones como verdad o representación (desde la epistemología de la Historia), como explica:

En las últimas décadas, la noción de representación (histórica) se ha discutido intensamente (...) La polémica ha sido intensamente útil para aclarar que la representación no es reductible a la verdad y que, en consecuencia, cuando el filósofo usa la verdad como único instrumento para explorar la relación semántica entre representación y el mundo, fracasa por necesidad (...) El principal resultado de mi análisis sobre la presencia ha sido que ella debe movernos más allá de la epistemología y, en consecuencia, ir más lejos del marco lógico dentro del cual todavía se mueven las teorías sobre la representación (Ankersmit, 2006: 171-172).

Como revelará más detalladamente en otro texto, Ankersmit prefiere hablar de "verdad representacional" (2006: 171-172), la cual no revela una verdad por correspondencia, sino una verdad intrincada al pasado que se hace "presencia". El problema es que son "sublimes", no estarían mediadas por el lenguaje ni por el sentido (Vergara, 2013: 239-249). El cómo nos relacionamos con el pasado, según Ankersmit, "escapa a la matriz intelectual de la verdad y la representación histórica" (2005: XIV), por ello es un fenómeno "sublime". En esa línea, reitera constantemente el carácter impráctico y especulativo de su propuesta. No existe en ella ningún atisbo de responder, siquiera discutir, el sentido histórico de escribir la Historia, por tanto, es una propuesta desvinculada de la matriz disciplinar expresada más arriba de la mano de Rüsen. Como acertadamente expresa la joven filósofa argentina María Inés La Greca (2013: 39), Ankersmit nos ofrece una visión de la escritura de la Historia "des-agenciada", que, según nuestra visión, corresponde a unos de los principales aportes desde una filosofía de la Historia post-narrativista.

Punto aparte merece la teoría y filosofía de la Historia que se está desarrollando en algunas partes de América Latina, en especial lo que se está generando en Brasil, México y Argentina a partir de la revista brasilera Historia da historiografia, los aportes desde México en la clásica revista Historia y Grafía, y el grupo Metahistoria y la recepción de la filosofía de la historia narrativista en Argentina. Para el primer caso nada más sirva mencionar la gran recepción de los trabajos de Rüsen en Brasil - en Chile poco y nada se conoce de su obra, lo que resulta vergonzoso y habla del estado de la teoría de la Historia en nuestro país -, la existencia de la Sociedad Brasilera de Teoría e Historia de la Historiografía (SBTHH) y la reciente puesta en línea del portal HH Magazine, lugar de encuentro de artículos, noticias, entrevistas y más, todos relacionados con teoría de la Historia.

El caso de México es también relevante. Historiadores como Guillermo Zermeño, el citado Alfonso Mendiola y Fernando Betancourt vienen hace ya muchos años aportando a la teorización de la disciplina. Desde una recepción sin parangón de la obra de Michel De Certeau - incluso mayor 
que en Francia - (o la traducción de nombres obligados como el mismo historiador jesuita, además de Hartog, Gumbrecht y otros), y de la teoría de sistemas de Niklas Luhmann, han salido a la luz textos recientes dignos de mencionar (Zermeño, 2018: 305-309), en especial la obra de Betancourt Historia y cognición: Una propuesta de epistemología desde la teoría de sistemas (UNAM, 2015), la cual es el primer intento por establecer una teoría y epistemología de la Historia desde una interpretación sistemática de la teoría de sistemas. Betancourt además tiene aportes teóricos notables desde la recepción de Freud y el psicoanálisis, De Certeau y Foucault. Cabe destacar desde México la existencia de la Red de Investigadores en Teoría y Metodología de la Historia (http://www.historiografia. enesmorelia.unam.mx/index.php/red-new ), instancia académica de la cual el responsable de este escrito forma parte.

En Argentina el grupo Metahistoria ha realizado interesantes aportes junto a la editorial Prometeo en la traducción de textos de autores como David Carr, White, Agamben y otros. Nombres como Verónica Tozzi, María Inés La Greca, Natalia Taccetta y Nicolás Lavagnino son obligados para una filosofía de la Historia actualizada que lamentablemente poco diálogo ha podido establecer con la historiografía local.

No está demás expresar que lo anterior es una tipología tentativa para efectos explicativos de un movimiento intelectual que se acelera y expande, complejo de observar desde un punto de vista holístico. Herman Paul, uno de los jóvenes teóricos de la Historia de alto impacto por estos años, podría ser mal encasillado en este tercer grupo, ya que su trabajo merece un punto a parte.

\section{Las relaciones con el pasado}

Paul trabaja, como hemos dicho, el oxímoron del pensamiento histórico, donde toda referencia al pasado está mediada por algún tipo de relación. El aporte de Paul radica en un marco teórico global para la teoría de la Historia donde no se descarta el trabajo epistémico, como sí lo hace Ankersmit desde su "experiencia sublime". Podría decirse que uno de los objetivos de Paul es llegar a conformar un sistema teórico general para la teoría de la Historia, tal como lo intenta Rüsen, pero desde un diálogo más fructífero con los narrativistas.

De todos los tipos de relaciones con el pasado que el historiador holandés propone (ver cuadro), la llamada "relación material" es aquella que, al no ser una relación de tipo teleológica, se configura no desde un telos, sino que desde aquello que le reclamamos al pasado, como explica Paul (2015a: 34): desde la "deuda y dependencia".

\begin{tabular}{|l|l|}
\hline \multicolumn{2}{|c|}{ Relaciones con el pasado: una tipología } \\
\hline Relación & Propósitos/apunta a... \\
\hline Epistémica & Conocimiento, comprensión \\
\hline Moral & Bien, justicia \\
\hline Política & Ejercicio de poder, toma de decisiones \\
\hline Estética & Belleza coherencia \\
\hline Material & \\
\hline
\end{tabular}


Paul construye una tipología de relaciones con el pasado donde hace notar que la sola relación en búsqueda de conocimiento (epistémica) no basta para dar cuenta de la pluralidad de fenómenos que se pueden observar dentro del pensamiento histórico, el cual, digámoslo en voz alta, no corresponde ni es un campo exclusivo de la disciplina. El aporte de Paul es dar cuenta de manera clara que la multiplicidad de relaciones que establecen los sujetos con el pasado se pueden centrar en estas cinco relaciones: epistémica, moral, política, estética, y material.

Coincidimos con el autor en que la relación de tipo material es la más problemática. Y esto debe ser explicado desde un punto de vista que Paul desliza, pero no profundiza: que las relaciones con el pasado son un conjunto de problemas temporales, y están regidas por la tensión entre lo que le pedimos y/o exigimos al pasado, en conjunto con lo que esperamos del futuro. Donde no podríamos estar en desacuerdo es en que, según nuestro autor, "la relación material precede a todas las demás", ya que "gira en torno a lo que el pasado hace a las personas y no a lo que las personas hacen con el pasado". Si bien el texto comentado es una herramienta orientadora, la teoría de la Historia en Paul debe ser complementada por los aportes de Rüsen, en la medida que encontramos en el pensador alemán las bases sistemáticas que nos permiten pensar esas "relaciones con el pasado". Allí radica una diferencia sustancial entre ambos teóricos: Paul se esfuerza por demostrar cierta distancia de las tesis más radicales en White y Ankersmit - a nuestro juicio, asunto que no logra, lo que se observa en la valoración de las relaciones estéticas por sobre las epistémicas -, mientras que Rüsen se posiciona en una vereda distinta, mucho más cercana a la posición de los aportes del filósofo Paul Ricoeur, en la perspectiva de una confianza en la teoría de correspondencia de la representación historiadora. Dicho de otro modo, en la perspectiva de Paul el problema del sentido y orientación del pasado hacia el futuro parece no ser tan importante como para Rüsen.

Ahora bien, a diferencia del pensamiento de Rüsen, Paul entrega mayor énfasis a las "relaciones" estéticas y narrativas con el pasado, en detrimento de las epistémicas, evidenciando su cercanía con el legado de White, como expresa:

"Los relatos históricos tienen pretensiones epistémicas e implicaciones morales o políticas, como veremos en los capítulos siguientes. Pero, ante todo, esos relatos tienen un aspecto estético: tienen una forma literaria que determina, al menos en parte, lo que el autor puede decir sobre el pasado" (Paul, 2015a: 68).

Rescatamos del trabajo de Paul la operatividad que para este escrito representa su tipología de "relaciones con el pasado", pero otra cosa es compartir con él la valoración que hace de las mismas al expresar que le resulta problemático hablar de ciertas "correspondencias con la realidad histórica", esperando que las discusiones historiográficas evidencien nada más que hipótesis consensuadas (2015b: 114), lo cual está muy cerca de la posición narrativista, donde no se espera una correspondencia entre presente y pasado de la investigación.

De todos modos, Paul acierta al panorama general de la teoría de la Historia entrado el siglo XXI, distinguiendo dos grandes marcos de trabajo en que hoy se mueve la teoría de la Historia: para aquellos que estudian el discurso historiográfico desde la filosofía de la ciencia, el objeto de estudio son los historiadores, donde cabrían posiciones como las de Tucker y Kuukkanen; y otra posición donde la mira está puesta sobre expresiones no académicas del pensamiento histórico, y de allí las citadas relaciones con el pasado. Ejemplo de ello es lo que Peter Seixas trabaja para la historia de la educación desde la conciencia histórica y lo que Rüsen viene trabajando desde la "cultura histórica". El objetivo de la teoría de la Historia hoy sería para Paul la capacidad de relacionar 
y hacer dialogar ambas posturas desde la idea de las "relaciones con el pasado" en la "cultura histórica", fenómeno que no le es propio sólo a los historiadores profesionales.

\section{Conclusiones}

Más allá de cualquier síntesis acerca de los derroteros de la teoría de la Historia en la actualidad - problema que siempre resultará algo antojadizo - lo cierto es que existe una problemática que atraviesa prácticamente el grueso de las discusiones actuales: la temporalidad. Para comprobarlo nada más habría que revisar el programa de las últimas jornadas de la International Network for Theory of History, realizadas en Estocolmo en agosto del 2018. Como habíamos dicho, los historiadores han llegado tarde a la discusión y los que lo han hecho, necesariamente han tenido que recurrir a debates teóricos y filosóficos previos. No es casualidad que un gran teórico de la historia como el alemán Lucian Hölscher fuera el autor de un libro esclarecedor al respecto: El descubrimiento del futuro (original de 1999). Antes, y por todos conocidos, son los aportes de Reinhart Koselleck desde la semántica histórica y la historicidad del tiempo humano, la posterior recepción de su teoría en la filosofía del lenguaje del Paul Ricoeur de Temps et récit III, y la posterior proyección de François Hartog con sus "regímenes de historicidad" desde un renovado estructuralismo.

Pasado y futuro se han convertido en problemas cada vez más comunes para pensar la historicidad de nuestro tiempo y la propia escritura de la Historia. Los aportes recientes de María Inés Mudrovcic (2013) desde la relación entre la historización de la memoria en los últimos años y los "órdenes del tiempo" de Hartog bajo la existencia de "regímenes historiográficos", o la relación fundante de la idea de futuro para comprender nuestro actual presentismo bajo la noción de "cambios sin precedentes" en los aportes de Simon (2018: 200-203), son ejemplos, entre otros, de los caminos que ha tomado la teoría de la Historia. Todos ellos, sea bajo la vereda que sea - más o menos cercanos al narrativismo postmoderno, (neo) positivismo moderno o hermenéutica posestructuralista -, apuntan la mirada hacia una teorización de la práctica historiográfica y de la cultura histórica, intentando dar nuevos bríos a la disciplina.

Ejemplo palpable es este dossier de la revista Autoctonía, por lo que solo nos queda aplaudir la iniciativa y agradecer la invitación a publicar de sus organizadores, esperando que en nuestro país se abran las puertas para un debate teórico siempre necesario. Este dossier, la pronta publicación en nuestro país del libro Representación histórica y nueva experiencia del tiempo, coordinado por Pablo Aravena (editorial América en Movimiento) - fruto del Primer Encuentro de Teoría de la Historia realizado en Santiago y Valparaíso en abril del 2018 -, y las periódicas Jornadas Internacionales de Teoría y Filosofía de la Historia, organizadas cada dos años por la Universidad Adolfo Ibáñez - que culminan en la publicación de un libro con las mejores ponencias - son claros ejemplos de que están las condiciones para que la teoría de la Historia tome posición en la academia chilena.

Este trabajo intenta ser un aporte desde ese "país de historiadores" que hasta ahora poco han querido reflexionar acerca de su propia práctica. Quizás el único historiador chileno - no dedicado a temas teóricos pero que sí se ha detenido en ello - que escapa a esta lamentable tendencia es el profesor Eduardo Cavieres $(2003,2009,2016)$, quien sí ha procurado reflexionar acerca de nuestra práctica más allá de simples reflexiones de la experiencia de investigación. Sirva una cita de su pluma para terminar este trabajo:

Quizás esta nueva época, para algunos la de la post-modernidad, pueda definirse como la de una especie de meta- 
ciencia (...) Ello ha llevado, nuevamente, a que la historia se restringa a las descripciones del pasado y olvide el papel de auto-revelación asignado por el propio Heródoto. Me parece que con respecto a la historia y la historiografía, se han venido produciendo algunos fenómenos de mucha importancia en lo que viene siendo una especie de des-conciencia o de alienación generalizada que impide el poder distinguir efectivamente lo verdaderamente trascendente que está sucediendo (Cavieres, 2009: 19).

\section{Bibliografía}

Ankersmit, F. (2005): Sublime Historical Experience, Stanford, Stanford University Press.

Ankersmit, F. (2006): "Representación, "presencia" y experiencia sublime", Historia y Grafía, N²7, pp. 171-172.

Betancourt Martínez, F. (2011): "¿Por qué es necesaria la investigación en teoría de la historia?", Históricas, No 90, pp. 16-21.

Bevernage, B. (2012): "From Philosophy of History to Philosophy of Historicities", Low Countries Historical Review, 127(4), pp. 113-114.

Bloch, M. (1952): Apologie pour l'histoire ou métier d'historien, París, Armand Colin.

Cavieres, E. (2003): “Reflexiones sobre la historia y la sociedad. Entre la conciencia del presente y el simple presentismo", Prólogo a Raíces de Expresión, Revista de los Estudiantes de Historia, Universidad Católica de Valparaíso, 2(2).

Cavieres, E. (2009): La Historia en controversia. Reflexiones, análisis, propuestas, Valparaíso, Ediciones Universitarias de Valparaíso.

Cavieres, E. (2016): "Las incertidumbres del tiempo en presente y la recuperación de la conciencia de ser", Historia 396, 6(1), pp. 61-74.

Dosse, F. (2012): El giro reflexivo de la historia. Recorridos epistemológicos y atención a las singularidades, Santiago, Ediciones Universidad Finis Terrae.
Ghosh, R.y Kleinberg, E. (2013): Presence. Philosophy, History, and Cultural Theory for the Twenty-First Century, Ithaca, Cornell University Press.

Kleinberg, E. (2017): Haunting History. For a Deconstructive Approach to the Pas, Stanford, Stanford University Press.

Kuukkanen, J. M. (2015): Postnarrativist. Philosophy of Historiography, Houndmills, Basingstoke, Hampshire, New York, Palgrave Macmillan.

La Greca, M. I. (2013): "Entre la ironía y el romance: Pasado, presente y futuro de la filosofía de la historia narrativista", Páginas de filosofía, 14(17), pp. 22-48.

Lorenz, C. (2017): "The Times They Are a-Changin", en M. Carretero, et.al., Time, Space and Periodization in History, Houndmills, Palgrave Handbook of Research in Historical Culture and Education, Palgrave.

Mendiola, A. (2000): "El giro historiográfico: la observación de observaciones del pasado", Historia y Grafía, № 15, pp. 181-208.

Mudrovcic, M. (2013): “Regímenes de historicidad y regímenes historiográficos: del pasado histórico al pasado reciente", Historiografías, Nº 5, pp. 11-31

Ovalle, D. (2017): “Paul Ricoeur y el pacto de verdad entre historiador y lector: epistemología y condición histórica", en P. Corti, J. L. Widow y R. Moreno, La verdad en la historia. Inventio, creatio, imaginatio, Santiago, RiL editores - Universidad Adolfo Ibáñez, pp. 75-90.

Ovalle, D. (2018): "Roger Chartier y Paul Ricoeur: Representación y verdad como fundamentos del trabajo historiador", Historia 396, 8(2), en prensa.

Paul, H. (2015a): Key Issues in Historical Theory, New York, Routledge.

Paul, H. (2015b): "Relations to the past: a research agenda for historical theorists", Rethinking History, 19(3), pp. 450-458.

Pihlainen, K. (2014): "The eternal return of reality: On constructivism and current historical desires", Storia della Storiografia, 65(1), pp. 103-115.

Runia, E. (2006): "Presence", History and Theory, Vol. 45, pp. 1-29.

Rüsen, J. (2013): Tiempo en ruptura, México, Universidad Autónoma Metropolitana Azcapotzalco. 
Simon, B. (2018): "History Begins in the Future: On Historical Sensibility in the Age of Technology", en S. Helgesson y J. Svenungsson (eds.), The Ethos of History: Time and Responsibility, New York, Berghahn.

Tamm, M. (2014): "Truth, Objectivity and Evidence in History Writing", Journal of the Philosophy of History, Vol. 8, pp. 265-290.

Tucker, A. (2004): Our Knowledge of the Past: A Philosophy of Historiography, Cambridge, Cambridge University Press.

Tucker, A. (2014): "Historical Truth", en V. Hösle, Forms of Truth and the Unity of Knowledge, Indiana, University of Notre Dame Press, pp. 232-259,

Vergara Anderson, L. (2013): "Nuevo Historicismo para el siglo XXI", Historia y Grafía, № 40, p. 239-249.

Recibido: 06 de diciembre de 2018

Aceptado: 05 de enero de 2019 